\title{
Clinical Efficacy of Three Different Electronic Apex Locators in Comparison with Radiographic Working Length Determination
}

\author{
Ayca Yilmaz, Selçuk Gokyay, Başak Gokyay, ilda Sinem Birdal and Enver Sedat \\ Kucukay
}

Department of Endodontics, Faculty of Dentistry, Istanbul University, Istanbul, Turkey

Correspondence should be addressed to: Ayca Yilmaz; aycasimsek@gmail.com

Received date: 29 July 2013, Accepted date: 30 January 2014; published date: 5 June 2015

Academic Editor: Ivana Miletić

Copyright (C) 2015. Ayca Yilmaz, Selçuk Gokyay, Başak Gokyay, ilda Sinem Birdal and Enver Sedat Kucukay . Distributed under Creative Commons CC-BY 4.0

\begin{abstract}
The aim of this study was to test the efficacy of three different electronic apex locators (EALs) in comparison with the radiographic working length determination. This study was conducted on the 211 root canals of 113 teeth in the undergraduate endodontic clinic. Under the referee of the research assistants, the students determined the working length by taking a periapical radiograph with the file in place initially and then electronically by use of three different apex locators. The research assistant observed the entire procedure based on the manufacturers' recommendations, and the measurements were recorded individually. Data were statistically analyzed using the NCSS-PASS 2007 program, one-way ANOVA and intraclass correlation coefficient at the $95 \%$ confidence level with significance set at $\mathrm{p}<0.05$. Comparing the coincidence between measurements obtained with radiography and with the three EALs, the intraclass correlation coefficient revealed that the radiographic and electronic working length measurements were coincident in all groups of teeth included in this study.
\end{abstract}

Keywords: electronic apex locators, Raypex4, ApexPointer, ProPex

\section{Introduction}

Accurate working length (WL) determination is a crucial factor for successful root canal treatment, reducing the chance of insufficient cleaning of the canals or damaging the periapical tissues from overinstrumentation (Sjögren et al., 1990; Ricucci and Langeland, 1998).

Several methods have been used to determine the $\mathrm{WL}$, such as radiographs, tactile sensation, and the presence of fluids on paper points. The radiographic method described by Ingle (2002) is one of the

Cite this Article as: Ayca Yilmaz, Selçuk Gokyay, Başak Gokyay, İlda Sinem Birdal and Enver Sedat Kucukay (2015)," Clinical Efficacy of Three Different Electronic Apex Locators in Comparison with Radiographic Working Length Determination", Journal of Research and Practice in Dentistry, Vol. 2015 (2015), Article ID 260644, DOI: 10.5171/2015.260644 
most common and reliable methods in determining the WL. However, the accuracy of this method is argumentative, because of the radiographical failures and the different locations of physiological foramina.

Custer (1918) described the first electronic device used for WL determination. Suzuki (1942) studied the use of direct current to measure canal lengths. Sunada (1962) demonstrated that the electrical resistance between the periodontal ligament and the oral mucosa had a constant value that could be measured.

Modern Electronic Apex Locators (EALs) use alternating current and detect changes in the impedance of the canal where the impedance is the ratio between the applied voltage and the resulting current in an alternating current electrical circuit (Venturi and Breschi, 2005). EALs determine the WL by measuring impedance with different frequencies between the file tip and the canal fluid. The impedance is the smallest at the apical constriction, and has a higher value at the major foramen (Gordon and Chandler, 2004).

ProPex (Dentsply Maillefer, Ballaigues, Switzerland) is a multi-frequency based on apex locator which uses multiple frequencies to determine the root canal length, and uses the energy of the signal for calculation, where the other apex locators usually use the amplitude of signal (Plotino et al., 2006). It was stated by the manufacturer that ProPex locates the apical foramen under any canal condition (wet, dry, sodium hypochlorite, etc) as a result of its multifrequency technology (Özsezer et al., 2007). The Apex Pointer (MicroMega, Besancon, France) is based on the calculation of the impedance difference at two frequencies (Nekoofar et al., 2006). Raypex4 (VDW, Munich, Germany) is also marketed as namely Bingo 1020 (Forum Engineering Technologies, Rishon Lezion, Israel) (Gordon \& Chandler 2004). It was reported by Gordon \& Chandler (2004) that this device uses two separate frequencies, and the manufacturers claim that the combination of using only one frequency at a time and basing measurements on the root mean square values of the signals increases the measurement accuracy and the reliability of the device.

Several in vivo and ex vivo studies have been conducted on various EALs to determine their accuracy and consistency (Fouad et al., 1990; Fouad et al., 1993; Frank and Torabinejad, 1993; Mayeda et al., 1993; Pallares and Faus, 1994; Dunlap et al., 1998; ElAyouti et al., 2002, Kaufman et al., 2002; Venturi and Breschi, 2005; Ozsezer et al., 2007; Comin Chiaramonti et al., 2012; Calıskan, 2013), and approximately $90 \%$ efficacy has been reported (Fouad et al., 1993; Frank and Torabinejad, 1993).

The aim of this clinical study was to test the efficacy of three different EALs in comparison with the radiographic WL determination.

\section{Materials and Methods}

This study was performed at Istanbul University, Faculty of Dentistry, Endodontics Department in the Undergraduate Students' Clinic under the referee of the endodontic research assistants. A total of 103 patients were included in this study. Patients using heart pacemakers were excluded. A standardized informed written consent was signed by each of the patients. This study was conducted on the 211 root canals of 113 teeth that would undergo endodontic treatment. Of the 113 teeth, 42 were incisors, 24 premolars and 47 molars; 61 were vital and 52 were non-vital.

A standardized periapical radiograph was taken from each tooth by using the extension cone paralleling technique instrument (XCP, Rinn Corporation, Illinois, USA). Roots with periapical radiolucencies and resorptions, fractures, open apices, or radiographically invisible canals were excluded from the study. The patients' profiles, chief complaint, medical and dental history, diagnosis, and vitality of the tooth as determined by bleeding on access and WL measurements were recorded. 
After administration of local anesthesia, the teeth were isolated with rubber dam, and the caries and metal restorations were removed. The access cavity was prepared in such a way that straight line access to the root canals was provided, and undercuts were avoided. The pulp status (vital or non-vital) was recorded according to the presence of bleeding in the entrance of the pulp chamber, and this was confirmed by the previous electronic vitality test records. The pulp chamber was cleaned, and the canal orifices were irrigated with $2.5 \%$ sodium hypochlorite (NaOCl) solution (Wizard, Rehber Chemistry, Istanbul, Turkey), and the access cavity was dried by aspiration of the solution.

No attempt was made to clean debris or pulp tissue remnants prior to introducing a size 15 K-file (Dentsply Maillefer, Ballaigues, Switzerland) into the canals, except irrigation. Then, a periapical radiograph with a size $15 \mathrm{~K}$-file within the root canal was taken to determine the WL. The distance from the reference point to the file tip that was located at 0.5 to $1 \mathrm{~mm}$ short of the radiographic apex was recorded as the WL.

Then, the students determined the WL electronically by the random use of three different apex locators such as Raypex4, ApexPointer, and ProPex. The EALs were used based on the manufacturers' recommendations. The lip clip was placed in the corner of the patient's mouth, and the file holder was attached to the shaft of the file. The size $15 \mathrm{~K}$-file was advanced apically into the canal, then the enlarged image of the apex zone was displayed, and the duration of the audio beeps was increased. As the apex was achieved, a constant tone appeared instead of beeps and a figure appeared at ' 0 ' point on the screen. The length of the K-file at this position was measured and $0.5 \mathrm{~mm}$ was subtracted from this measurement according to the manufacturers' recommendations. The individual measurements were recorded on each of the patient's chart.

The data were then statistically analyzed using the NCSS-PASS 2007 program, oneway ANOVA, and intraclass correlation coefficient at the $95 \%$ confidence level with significance set at $\mathrm{p}<0.05$.

\section{Results}

Comparing the coincidence between the radiographic and the three electronic measurements, the intraclass correlation coefficient at the $95 \%$ confidence level revealed that the radiographic and electronic WL measurements were coincident in all of the root canals included in this study (Radiography vs. ProPex 0.995, Radiography vs. ApexPointer 0.994, Radiography vs. Raypex4 0.995) (Table 1). The overall mean and standard deviations calculated for radiography $19.70 \pm 2.14$, ProPex 19.71 \pm 2.14 , Apex Pointer

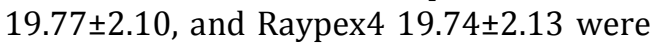
evaluated and no statistically significant difference was detected among the groups $(\mathrm{p}=0.984, \mathrm{p}=0.985)$.

Table 1: Comparison of the radiographic and the EALs' measurements by the intraclass correlation coefficient at the $95 \%$ confidence level

\begin{tabular}{|l|l|}
\hline Overall Groups & ICC 95\% IC \\
\hline Radiography vs ProPex & $0,995(0,991-0,997)$ \\
\hline Radiography vs Apex Pointer & $0,994(0,990-0,997)$ \\
\hline Radiography vs Raypex4 & $0,995(0,991-0,997)$ \\
\hline
\end{tabular}


Statistical analysis showed that the radiographic and the EAL measurements were coincident for all of the incisor, premolar and molar groups, and the difference was found to be insignificant (Table 2).

Table 2: Comparison of the radiographic and the EALs' measurements for all tooth groups (incisors, premolars and molars) by the intraclass correlation Coefficient at the $95 \%$ confidence level

\begin{tabular}{|l|l|l|l|}
\hline vital+non-vital & Incisors (n:42) & Premolars (n:34) & Molars (n:135) \\
\hline Radiography vs ProPex & $0,995(0,991-$ & $0,988(0,977-$ & $0,947(0,926-$ \\
& $0,997)$ & $0,988)$ & $0,962)$ \\
\hline Radiography vs Apex & $0,994(0,990-$ & $0,983(0,967-$ & $0,913(0,878-$ \\
Pointer & $0,997)$ & $0,991)$ & $0,938)$ \\
\hline Radiography vs Raypex4 & $0,995(0,991-$ & $0,990(0,980-$ & $0,951(0,932-$ \\
& $0,997)$ & $0,995)$ & $0,965)$ \\
\hline
\end{tabular}

With regard to the vitality status as vital vs. non-vital, the radiographic and EAL measurements in all tooth groups were found to be statistically coincident as well.

\section{Discussion}

Root canal preparation should ideally be performed to the cementodentinal junction or the apical constriction (Ricucci and Langeland, 1998; Gordon and Chandler, 2004). Root canals that were over or under prepared and filled may decrease the success rate of endodontic therapy (Seltzer et al., 1973). Kuttler (1955) showed that when a file tip is $1 \mathrm{~mm}$ short of the anatomic apex, it will be within a range at which the apical constriction is generally located. However, it is appropriate to subtract $0.5 \mathrm{~mm}$ from the radiological root canal length to calculate the $\mathrm{WL}$ as suggested by several studies (McDonald and Hovland, 1990; Weine, 1996). In this study, the students were instructed to calculate the WL 0.5 to $1 \mathrm{~mm}$ short of the radiographic apex, as well.

The use of electronic devices to determine the WL has gained increasing popularity in recent years. EALs have advantages of easy and fast application, reduction of exposure to radiation, detection of perforations, safe use in pregnancy in patients with vomiting reflex, and mentally retarded patients. In the previous studies that have been conducted to test the accuracy and efficacy of different brands of EALs, it has been mostly reported that the electronic method revealed $79 \%$ to $94 \%$ accuracy, depending on the method of comparison (Katz et al., 1991; Frank and Torabinejad, 1993; Mayeda et al., 1993; Pallares and Faus, 1994). The Bingo 1020 and Propex apex locators were found to be equally accurate in positioning the file at $\pm 0.5 \mathrm{~mm}$ of the root apex, and provide reliable measurements in calculating the WL (Comin Chiaramonti et al., 2012). Kaufman et al. (2002) concluded that both EALs, Bingo 1020 and Root ZX (J. Morita Corp., Tustin, California), used in an in vitro study measured the tooth length with great accuracy and a significant positive correlation was found between the two devices. No significant difference was found between the two apex locators when measurements were taken with different irrigants and the content of the root canal did not affect the accuracy of the measurements. Caliskan et al. (2013) evaluated clinically the accuracy of two EALs in determining the position of the apical foramen of teeth with large periapical lesions and persistent intracanal exudates, and concluded that ProPex and Apex Pointer determined the position of the major apical foramen with a high degree of accuracy with no significant differences between them. The EALs, ProPex, ApexPointer and Raypex4 used in the present study revealed that the WL measurements were reliable and in coincidence with the radiographic measurements, similar to the findings of 
the aforementioned studies using somehow the same EALs and reporting their reliability and accuracy tested clinically or experimentally.

Some clinical studies evaluating the efficacy of several EALs on vital and non-vital teeth reported that the pulp vitality had no significant effect on the accuracy of electronic WL measurements (Fouad et al., 1990; Fouad et al., 1993; Dunlap, 1998; Venturi and Breschi, 2005). The results of the present study were in accordance with the aforementioned studies showing that the radiographic and EAL WL measurements were coincident in all of the tooth groups regardless of the vitality status. However, it was suggested that in non-vital cases with inflammatory root resorption, the apical constriction might be altered, which would lead to a lower accuracy (Pommer et al., 2002). Since the root canals associated with periapical radiolucencies and resorption were excluded from this study, the results were not significantly influenced by the pulp status.

The lonely use of EALs without the preoperative and postoperative radiographs is not recommended in clinical practice due to the large variations in tooth morphology and to the medico-legal record keeping requirements (Gordon and Chandler, 2004). The combined use of radiography and EAL for WL determination has resulted in greater accuracy (ElAyouti et al., 2002). As was stated in a case report; when pre- or post-radiographs were not taken from the pregnant patient, healing was not achieved due to a missed and untreated root canal (Segura-Egea et al., 2002). However, it has also been suggested that the correct use of an EAL alone could prevent the need for further diagnostic radiography for determination of $\mathrm{WL}$ (Smadi, 2006).

The results of this study revealed that the overall radiographic and EAL measurements were coincident in both vital and non-vital teeth. However, these results should be interpreted within the parameters of this study, and the varying degree of clinical conditions must be taken into consideration for the need of the combined use of radiography and EAL for WL determination.

\section{Conclusions}

Under the clinical conditions of this study, it is revealed that the proper use of EALs can be considered and coincides with radiography in WL determination.

\section{References}

1. Caliskan, M., Kaval, M. and Tekin, U. (2013) 'Clinical accuracy of two electronic apex locators in teeth with large periapical lesions,' Int Endod J, 19 (in press).

2. Comin Chiaramonti, L., Menini, M. and Cavalleri, G. (2012) 'A comparison between two fourth generation apex locators,' Minerva Stomatol, 61(5):183-96.

3. Custer, C.(1918) 'Exact methods for locating the apical foramen,' J Nat Dent Assoc, 5: 815-19.

4. Dunlap, C.A., Remeikis, N.A., BeGole, E.A. and Rauschenberger, CR. (1998) 'An in vivo evaluation of an electronic apex locator that uses the ratio method in vital and necrotic canals,' J Endod, 24: 48-50.

5. ElAyouti, A., Weiger, R. and Löst, C. (2002) 'The ability of Root ZX apex locator to reduce the frequency of overestimated radiographic working length,' J Endod, 28: 116-9.

6. Fouad, A.F., Krell, K.V., McKendry, D.J., Koorbusch, G.F. and Olson, RA. (1990) 'A clinical evaluation of five electronic rootcanal length measuring instruments,' J Endod, 16: 446-9.

7. Fouad, A.F., Rivera, E.M. and Krell, K.V. (1993) 'Accuracy of the Endex with variations in canal irrigants and foramen size,' J Endod, 19: 63-7.

8. Frank, A.L. and Torabinejad, M.(1993) 'An in vivo evaluation of Endex electronic apex locator,' J Endod, 19: 177-9. 
9. Gordon, M.P.J. and Chandler, N.P. (2004) 'Electronic apex locators,' Int Endod J, 37: 425-37.

10.Ingle, J.I. and Backland, L.K.(2002) Endodontics, 5th ed., Elsevier, Canada.

11.Katz, A., Tamse, A. and Kaufman, Y. (1991) 'Tooth length determination: A review,' Oral Surg Oral Med Oral Pathol, 72: 238-42.

12.Kaufman, A.Y., Keila, S. and Yoshpe, M. (2002) 'Accuracy of a new apex locator: an in vitro study,' Int Endod J, 35(2): 186-92.

13.Kuttler, Y. (1955) 'Microscopic investigation of root apexes,' J Am Dent Assoc, 50: 544-52.

14.Mayeda, D.L., Simon, J.H., Aimar, D.F. and Finley, K. (1993) 'In vivo measurement accuracy in vital and necrotic canals with the Endex apex locator,' J Endod, 19: 545-8.

15.McDonald, N.J. and Hovland, E.J. (1990) 'An evaluation of the apex locator Endocater,' J Endod, 16: 5-8.

16.Ozsezer E, Inan U, Aydın U. 'In vivo evaluation of ProPex electronic apex locator.' J Endod. 2007 33(8): 974-7.

17.Pallares, A. and Faus, V. (1994) 'An invivo comparative study of two apex locators,' J Endod, 20: 576-9.

18.Pommer, O., Stamm, O. and Attin, T. (2002) 'Influence of the canal contents on the electrical assisted determination of the length of root canals,' J Endod, 28: 83-5.

19.Ricucci, D. and Langeland, K. (1998) 'Apical limit of root canal instrumentation and obturation. Part II: A histological study,' Int Endod J, 31: 394-409.

20.Segura-Egea, J.J., Jimenez-Pinzon, A. and Rios-Santos J.V. (2002) 'Endodontic therapy in a three rooted mandibular first molar: Importance of a through radiographic examination,' J Can Dent Assoc, 68: 541-4.

21.Seltzer, S., Soltanoff, W. and Smith, J. (1973) 'Biologic aspects of endodontics. V. Periapical tissue reactions to root canal instrumentation beyond the apex and root canal fillings short of and beyond the apex,' Oral Surg Oral Med Oral Pathol, 36: 725-37.

22.Sjögren, U., Hagglund, B., Sundqvist, G. and Wing, K. (1990) 'Factors affecting the long-term results of endodontic treatment.' J Endod, 16: 498-504.

23.Smadi, L. (2006) 'Comparison between two methods of working length determination and its effect on radiographic extent of root canal filling: $A$ clinical study,' BMC Oral Health, 6: 4.

24.Sunada, I. (1962) 'New method for measuring the length of the root canal,' $J$ Dent Res, 41: 375-87.

25.Suzuki, K. (1942) 'Experimental study on iontophoresis,' Jap J Stomatol, 16: 41129.

26.Venturi, M. and Breschi, L. (2005) 'A comparison between two electronic apex locators: An in vivo investigation,' Int Endod J, 38: 36-45.

27.Weine, F.S. (1996) Endodontic therapy, 5 th ed., Mosby, St Louis. 